Der Vorsitzende schloss hierauf die Jahresversammlung, welche als eine der erfolgreichsten und unterhaltendsten Vereinigungen den Teilnehmern in Erinnerung bleiben wird.

\title{
Über meine erfolgreichen Zuchten der Hakengimpel (Corythus enucleator) in der Gefangenschaft.
}

Vortrag gehalten am 29. Mai 1897 in Dresden.

Von Major A lexander v. Homeyer.

Als ich im Herbst 1891 die Waldjagden bei Herrn von Homeyer-Ranzin (Neu-Vorpommern) mitmachte, sagte mir Förster Schmidt-Zinnowitz (Insel Usedom), dass sich bei $\mathrm{ihm}$ in den Dohnen mehrere Karmingimpel gefangen hätten. Ich stellte sogleich fest, dass dies nur Hakengimpel sein könnten. Nach einiger Zeit teilte mir Förster Schmidt mit, dass er im Besitz von zwei lebenden Hakengimpeln sei. Noch selbigen Tags fuhr ich über Wolgast nach Zinnowitz, holte die Vögel - 2 alte rote Männchen - und kam Nachts $1 / 212$ mit denselben nach Greifswald zurück.

Im Laufe des Spätherbstes erschienen die Hakengimpel auch massenhaft auf dem Festlande, plünderten die Ebereschen und zogen weiter nach Süden. Bei dieser Gelegenheit bekam ich noch zwei lebende Weibchen und ein junges Männchen. Die Flüge bestanden grösstenteils aus rötlichen Vögeln, also Männchen, während Weibchen viel sparsamer waren. Somit hatte ich 2 Paare und 1 Reserve-Männchen.

Aus den betreffenden Zeitschriften über Vogelzucht brachte ich in Erfahrung, dass die Stubenvogel-Züchter mit der Hakengimpelzucht bis jetzt keine glücklichen Resultate erzielt hätten. Im glücklichsten Fall seien Eier gelegt und bebrütet, auch Junge erzielt worden, aber diese Jungen seien nach 2-3 Tagen gestorben. Diesem Misserfolg stand ich nun gegenüber, der ich niemals Vogelzucht betrieben hatte, denn als Offizier hatte ich dazu keine Gelegenheit gehabt. Dennoch beschloss ich, die Zucht zu wagen. - Da die kleinen in der Gefangenschaft erzielten Jungen immer bereits nach ein paar Tagen eingegangen waren, musste falsches Futter die Ursache dazu sein. Ich dachte darüber nach, was wohl als Futter zu reichen sei. Es war mir bekannt, dass die Hakengimpel im Hochnorden, sowohl in Norwegen-Schweden-Finland, Nord-Russland wie Sibirien ihr Brutgeschäft in grossen Nadelholz-Waldungen vollbringen, und somit kam mir der Gedanke, dass zur glücklichen Aufzucht der Jungen wohl Bestandteile der Coniferen (Kiefer oder Zirbelnuss) notwendig seien. - Der Gedanke, dass er gut und richtig sei, wurzelte sich immer fester, und somit beschloss ich, die Zucht zu wagen. - Es wurde eine grosse Volière angefertigt $\left(2^{1 / 2} \mathrm{~m}\right.$ lang, $1 \frac{1}{2} \mathrm{~m}$ hoch und $1 \frac{1}{2} \mathrm{~m}$ breit), diese mit Sitzstangen ver- 
sehen und inwendig mit kleinen Kieferzweigen besteckt. Auf dieselbe Weise richtete ich einen grossen Käfig her $\left(1 \frac{1}{4} \mathrm{~m}\right.$ lang, $3 / 4 \mathrm{~m}$ hoch, $3 / 4 \mathrm{~m}$ breit). Ich halte seit 45 Jahren viele Stubenvögel, besonders Insektenfresser (namentlich Sylvien und Drosseln), und ich liebe "grosse Käfige“, worin sich meine Vögel sehr wohl befinden und "sich auch trotz der Grösse des Gebauers" zurechtfinden, was seiner Zeit von einem Wiener Vogelhändler bestritten wurde. - Also ich setzte bald nach Weihnachten die beiden Hakengimpelpaare in die Volière. Bald sah ich, wie sich die Vögel ,paarweise“ gruppierten. Nun nahm ich das eine Paar aus der Volière heraus und that es in den grossen Käfig.

Als die Vögel Ende April Miene machten, sich zu begatten, that ich in die Volière eine hübsche sperrige Kiefer von $1 \frac{1}{2} \mathrm{~m}$ Höhe und in den Käfig einen hübschen dickbuschigen Kiefernast. Auf beide befestigte ich je ein Nest der Wachholder- resp. Singdrossel und arrangierte das Ganze möglicht naturgetreu, d. $h$. so, wie ich mir ein Hakengimpelnest im Freien vorstellte.

Der Anfang war gemacht, aber - ich musste fort, - fort nach Ungarn, wo ich 1891 Herrn Benedikt von Meszleny-Velencze meinen Besuch zugesagt hatte. Als alter Junggeselle instruierte ich meine Wirtschafterin, die fast 70jährige Frau Miltz geeignet und reiste ab. Meine Reise führte mich an den Velenczer-See zu Herrn v. Meszleny, an den Platten-See zu Herrn Rat v. Deiningen in Kaszthely, zu Herrn Vicegespan von Csató nach Nagy Enyed in Siebenbürgen und nach Bázias an der Donau zu Herrn Lindauer. Mit Letzterem durchkreuzte ich über Anina die SO. Karpathen und kehrte nach Bázias wieder zurück. - Hier traf mich am 26. Juni die briefliche Nachricht, dass daheim junge Hakengimpel in dem Neste der Volière seien und sich sehr wohl befänden. - Denselben Abend gaben mir Familie Lindauer in den Bergen in Gottes freier Natur ein Abschiedsessen, und den andern Morgen $3 / 44 \mathrm{Uhr}$ fuhr ich auf der Bahn der pommerschen Heimat $\mathrm{zu}$, die ich nach gut 48 Stunden erreichte, um nun die Hakengimpelzucht selbst in die Hand zu nehmen. Es war für mich ein wohliges Gefühl, die 4 Kleinen im Nest zu sehen, wie sie von beiden Alten gefüttert wurden. Aus der zweiten Hecke wurde nichts, doch lieferte sie mir eine grosse Zahl prächtiger Eier für meine oologische Sammlung.

Ich habe nun alle Jahre, also 1892 bis inkl. 96 (und auch noch nach meinem Vortrage in Dresden 1897) junge Hakengimpel mit mehr oder minder günstigem Erfolg gezüchtet, „a u ch mit Jungen, die bei mir geboren waren". Somit habe ich das Problem der Hakengimpelzucht gelöst. Ich habe 1892 über die erste gelungene Zucht in der Gefiederten Welt des Herrn Dr. Russ ausführlich Bericht erstattet. Soviel ich hörte, fand sich im Herbst 1892 auch ganz unerwarteter Weise ein junger Hakengimpel in der grossen Volière eines Züchters in Posen vor; ferner züchtete im Jahre 93 oder 94 ein Arzt in Breslau zwei 
junge Hakengimpel, der sich vorher bei mir erkundigte, wie man es anfangen müsse. Sonst habe ich von weiteren Erfolgen nichts gehört. -

Nun wird es aber wohl Zeit, dass ich einiges Nähere angebe, wie ich verfahren bin. - Ich füttere die Alten mit Hanfsamen und Raps, wozu ich etwas Mohn, Leinsamen, Hafer und Hirse gebe. Ausserdem bekommen meine Vögel Sonnenblumenkerne und - das ganze Jahr einen Napf Weichfutter ( $1 / 3$ Ameisenpuppen, $1 / 3$ Mohrrüben, $1 / 3$ säurefreies Eierbrod.) - Bei diesem Futter halten sich die Hakengimpel auf die Dauer, d. h. jahrelang, doch da die Vögel trotz ihrer Grösse und Stärke sehr leicht kümmern, so gebe ich das ganze Jahr jedem Vogel täglich 2-3 Mehlwürmer, und je nach der Jahreszeit allerlei Erfrischung, wie Vogelmier (namentlich mit Samenkapseln), Salat, Birnen, Pflaumen, Heidelbeeren, süsse Aepfel, Ebereschen, Hollunderbeeren etc. Es ist dies etwas umständlich, aber gut. Von Weihnachten ab stecke ich alle paar Tage frische Kiefernzweige in die Volière resp. Käfige. Die Vögel fressen sehr gern den Neuausschlag vorn an den Zweigen (die harzigen Neubildungen für nächstjährige Zweige). Kann ich es haben, so gebe ich zwischen das Körnerfutter einen starken Zusatz von Kiefersamen. Nähert sich die Brutzeit, so habe ich bei vorstehendem Futter äusserst gesunde und kräftige Vögel. Es erfolgt dann auch leicht die Begattung und das Eierlegen. Sind die Vögel nicht in guter Verfassung, so giebt es Verdruss, indem das Weibchen die Eier nicht in das Nest legt, sondern im Käfig fallen lässt. Ich bemerke hierbei noch, dass das Nest (Drosselnest) mit feinem Draht absolut fest gebunden sein muss; schwankt dasselbe, so wird nichts aus dem Brüten. Mit künstlichen Nestern, wie man sich bei der Kanarienvogelzucht bedient, hatte ich Misserfolge, auch nachdem ich ,grössere Nester" machen liess und diese ausfütterte. Man muss Drosselnester haben, die ja leicht zu beschaffen sind (Singdrossel). Es genügen solche, die man im Spätsommer aufsucht. Das Bautalent des Hakengimpels ist ,in der Gefangenschaft" sehr unbedeutend. Er legt in die Drosselnester auch kaum etwas zur Ausfütterung. -

Brütet nun das Weibchen stark, - die Jungen kommen den 13. oder 14. Tag - so sorge man durchaus für frische Kieferzweige, die man alle 2-3 Tage frisch reichen muss, und gebe zum Misch-Weichfutter viel gehacktes Hühnerei (hart gekocht). Dieses Ei-Mischfutter muss täglich 2 mal ,frisch" gereicht werden. Ausserdem Vogelmier, das in Samen getreten ist. Kommen nun die Jungen, so ist alles in Ordnung, die Alten - wenn sie gut sind - füttern mit Leichtigkeit die Kleinen auf, - müssen nun aber zu all' dem gereichten Futter noch viele Mehlwürmer haben. Ich reichte täglich 3 mal je 25-30 Mehlwürmer für eine Brut von 4 Jungen. Mit frischen Ameisenpuppen geht die Aufzucht jedenfalls leichter und weniger mühsam. Ich habe das Füttern 
viel beobachtet. Der Alte oder das Weibchen nehmen Hanfsamen, dann Mischfutter mit Ei, gehen dann an die Kiefernzweige und nagen davon vorn von den Herzschösslingen, nehmen 2-3 Mehlwürmer, den weichen Inhalt auslutschend (der Kopf und die Haut werden fallen gelassen), dann geht es an das Grünfutter (Mier) und dies alles wird im Kropf richtig gemischt und bearbeitet und erst dann - es vergehen Minuten - den Jungen gereicht.

Das Geheimnis der glücklichen Zucht liegt meines Erachtens in der Beimischung von Herzteilen der Coniferen und dem Geben von vielen Mehlwürmern. - Die Mehlwürmer sind unentbehrlich, denn unsere Körnerfresser füttern in freier Natur ihre Jungen viel mehr mit animalischem wie vegetabilischem Nahrstoff, namentlich in der ersten Zeit.

Aus all' diesem ersieht man, dass es nicht Jedermanns Sache ist, Hakengimpel aufzuziehen, sie bedürfen ganz ausserordentlich vieler Mühe und Aufmerksamkeit des Züchters, sonst sind Misserfolge in Masse vorhanden. Jede Vernachlässigung im Futterreichen rächt sich sofort. Ich habe erlebt, dass 3 Junge schon so gross waren, dass sie fast schon allein fressen konnten, während die Alten schon wieder an neue Brut dachten und das alte Männchen zu füttern aufhörte. Das Weibchen aber fütterte nur 2 Junge, so dass das grösste verhungerte. Ich wollte es stopfen, aber da ich kein Kropffutter geben konnte, so trat bald der Tod ein. Dann fütterte das Weibchen nur noch ein Junges, so dass das zweite auch starb. Inzwischen fand Begattung und Eiablage statt und auch das dritte Junge verhungerte. - Und ich sass dabei und konnte nicht helfen, die Jungen schrieen vor Hunger, und die Eltern fütterten nicht. Ich war ein paar Mal so in Wut, dass ich die Alten am liebsten gegen die Wand geworfen hätte. Erwähnen möchte ich noch, dass bei meiner Abwesenheit (Reisen) meine jetzige Wirtschafterin Frau Zahn selbständig 1895 und 1897 junge Hakengimpel gezüchtet hat.

Als Zimmervogel ist der Hakengimpel zu empfehlen. $\mathrm{Er}$ ist ein gemütlicher Vogel, der gegen seinen Pfleger bald alle Scheu ablegt. Ich muss dabei bemerken, dass die in den Käfig gebrachten Vögel ,anfangs ganz zahm" sind, dann aber "scheu“ werden und nun erst wieder zahm gemacht werden müssen. Die Vögel vertragen unsere Sommerwärme ganz gut, verlangen aber viel frisches Wasser und reine Luft, weshalb man die Fenster oft öffnen muss. Bei dicker Stubenluft gehen sie leicht ein. Der Gesang ist gerade nicht bedeutend, doch sehr volltönig und wohllautend, im Ton wie bei Carpodacus erythrinus. Die Hauptstrophe ist ein langes wellenförmiges Leyern, laut beginnend und leise endend. Man wird dabei an den weichen Lull-Gesang der Heidelerche (Lullula arborea) erinnert. Besonders schön nimmt sich der Hakengimpelgesang Nachts aus. Fast all unsere nordischen 
Vögel singen gern Nachts beim Vollmondschein. Gern erinnere ich mich dieser Concerte, die nicht übel sind, wenn in den verschiedenen Zimmern 5-8 Hakengimpelmännchen leiern, und sich dazu die hellaufjauchsenden Lockrufe des Spornammers (Calcarius lapponicus) gesellen, und der sibirische Weidenammer (Emberiza aureola) seine ortolanartigen, wehmütigen Strophen mit einmischt.

\section{Über die Vogelfauna des Südpolargebietes.}

Vortrag gehalten am 29. Mai 1897 zu Dresden von Herman Schalow.

(Im Auszuge mitgeteilt.)

Die Forschungen im Nordpolargebiet während der letzten fünfzig Jahre haben die Fragen, an deren Aufhellung man seit den Tagen Sir Hugh Willoughby's, also seit mehr denn drei Jahrhunderten, unermüdlich und mit dem Einsetzen aller menschlichen Energie thätig gewesen ist, soweit geklärt, dass die wichtigsten Probleme, welche die arktische Zone bietet, der endlichen Lösung nahe geführt betrachtet werden dürfen. Anders ist es im Gebiet des antarktischen Pols. Während fast alle Teile unseres Erdballes, den weitesten Umrissen nach, als durchforscht bezeichnet werden dürfen, bilden die Südpolarländer in dieser Richtung allein eine Ausnahme. Denn was wir in wissenschaftlicher Hinsicht von diesen, räumlich ungeheuren Teilen der südlichen Zone kennen, ist so ausserordentlich gering und dürftig, dass es dringend nunmehr an der Zeit erscheint, mit allen Mitteln, welche die Wissenschaft unserer Tage dem Forscher zur Verfügung zu stellen vermag, die endliche Erschliessung der Antarktis anzubahren.

Von diesem Gedanken durchdrungen fasste der XI. Deutsche Geographen-Tag, welcher im April 1895 zu Bremen seine Tagung hielt, den Beschluss, ,in voller Würdigung der Wichtigkeit der antarktischen Forschung für Geographie und Naturwissenschaft einen Ausschuss zu ernennen, dessen Aufgabe es ist, über die Möglichkeit einer baldigen Entsendung einer Deutschen wissenschaftlichen Expedition in die Antarktis zu beraten und günstigen Falles die Ausführung der Sache in die Wege zu leiten." Der im August desselben Jahres in London tagende VI. Internationale Geographen-Congress machte die Angelegenheit zu einer internationalen und empfahl, , in Anbetracht der aus der Erforschung der Antarktis voraussichtlich für alle Zweige der Wissenschaft sich ergebenden Vorteile, dass die verschiedenen gelehrten Gesellschaften der ganzen Welt auf dem ihnen am wirksamsten erscheinenden Wege darnach trachten, diese Aufgabe vor Ablauf des 19. Jahrhunderts gelöst zu sehen." Denn nicht allein für die specielle Geographie, so wurde bei den Verhandlungen in London des weiteren ausgeführt, nein für alle Disciplinen der Natur- 


\section{$2 \mathrm{BHL}$ Biodiversity Heritage Library}

1897. "Über meine erfolgreichen Zuchten der Hakengimpel (Corythus enucleator) in der Gefangenschaft." Journal fu

r Ornithologie 45, 520-524. https://doi.org/10.1007/bf02207250.

View This Item Online: https://www.biodiversitylibrary.org/item/103562

DOI: https://doi.org/10.1007/bf02207250

Permalink: https://www.biodiversitylibrary.org/partpdf/142547

\section{Holding Institution}

Smithsonian Libraries

\section{Sponsored by}

Biodiversity Heritage Library

\section{Copyright \& Reuse}

Copyright Status: Public domain. The BHL considers that this work is no longer under copyright protection.

This document was created from content at the Biodiversity Heritage Library, the world's largest open access digital library for biodiversity literature and archives. Visit BHL at https://www.biodiversitylibrary.org. 